\title{
Mejoras Productivas en el Tratamiento de Paja Toquilla en el Centro de Tratamiento de Paja Toquilla de la Comuna Barcelona, Parroquia Manglaralto, Provincia de Santa Elena, Ecuador.
}

\author{
Jorge Lucín Borbor ${ }^{1}$ \\ Facultad de Ingeniería Industrial. Escuela de Ingeniería Industrial \\ ${ }^{1}$ Universidad Estatal Península de Santa Elena (UPSE) \\ Campus La Libertad, vía principal Santa Elena - La Libertad \\ La Libertad-Ecuador \\ jorge lucin@yahoo.com
}

\begin{abstract}
Resumen
La comuna de Barcelona, parroquia Manglaralto del Cantón Santa Elena, es conocida por su tratamiento a la planta paja toquilla (Carludovicapalmata). En sus hectáreas de terreno en las montañas que colinda con la cordillera Chongón-Colonche, crece este vegetal en forma silvestre que es cosechado en aproximadamente tres años de cultivo. En el poblado, las familias lideradas por mujeres trabajadoras, realiza el tratamiento de la planta, por procesos de limpieza, cocción, secado y embalaje, que será destinado a sectores del país en donde elaboran los famosos sombreros de paja toquilla. Con el fin de mejorar el proceso de este tratamiento, el grupo NOBIS, el Consejo Provincial de Santa Elena y PORAMÉRICA, firmaron un convenio de cooperación que ha logrado conseguir los objetivos planteados. El proceso de secado, que constituía un problema en épocas de ausencia de sol, ha sido solucionado con la instalación de secadores industriales y a la vez, la cocción marginó el proceso de hacerlo con madero y leña, logrando eficiencia en este proceso con la utilización de quemadores con gas industrial, disminuyendo el impacto ambiental. El proceso administrativo mejoró con la implementación de documentos y control del proceso de producción y costos.
\end{abstract}

Palabras claves: mejoramiento, producción, convenio, paja toquilla, secado.

\begin{abstract}
The community of Barcelona, parish ManglaraltoCantón Santa Elena, is known for its treatment of the straw shawl floor (Carludovicapalmata). In its acres of land in the mountains adjacent to the Chongón-Colonche range, this plant grows wild it is harvested in about three years of cultivation. In the village, families headed by women workers, performing the treatment plant for cleaning, cooking, drying and packaging processes, which will be directed to areas in the country where they make the famous Panama hats. In order to improve the process of this treatment, the NOBS group, The Provincial Council of Santa Elena and PORAMÉRICA, have signed a cooperation agreement that has managed to achieve the objectives. The drying process, which was a problem in times of lack of sun, has been solved with installation of industrial dryers and simultaneously marginalized cooking process and do it with wood stove, achieving efficiency in this process with the use of burners with industrial gas, lowering environmental impact. The administrative process improved with the implementation of documents and control of the production process and costs.
\end{abstract}

Keywords: Improving, production, convention, straw shawl, dried. 


\section{Introducción}

Cuando hablamos de los sistemas de trabajo y trasformación de materia prima, nos referimos a empresas productivas de bienes para el consumo de la comunidad en general. Estos procesos de producción clasificados en 4 grandes grupos: producción por procesos, producción por componente fijo, producción de proceso continuolínea y producción mixta tiene sus derivaciones en función de la naturaleza de la transformación.

La Comuna Barcelona y el trabajo del CENTRO DE TRATAMIENTO DE PAJA TOQUILLA, es un sistema de trabajo por procesos que tradicionalmente ha sido artesanal y que busca la manera de mejorar en todas sus formas, estos es, infraestructura, sistemas de producción, calidad, ventas y recursos humanos, rentabilidad. $\mathrm{La}$ gestión que realizan los directivos de esta unidad de producción, mayoritariamente mujeres, radica en buscar el financiamiento para mejoras de esos aspectos. La firma de un convenio tripartito entre el grupo NOBIS, el Consejo Provincial de Santa Elena y PORAMÉRICA posibilita que se mejoren los procesos de cocinado, secado, gestión administrativa y de costos del tratamiento de paja toquilla. Es importante el aporte del convenio y su ejecución porque, el proceso de cocción tiene un cambio total al desechar la primaria actividad de cortar árboles, y convertirla en leña como combustible generando una contaminación al entorno, para ir a la utilización de gas licuado de petróleo, GLP, minimizando esta contaminación. El secado, que habitualmente se hace al medio ambiente tiene una alternativa inmediata en secadores industriales y con ello optimizando el tiempo de esta actividad. A más de ello, esto concatena el hecho de complementar estas mejoras con procesos de capacitación en desenvolvimiento administrativo y cálculos actualizados de costos y gastos para trasparentar la rentabilidad de este trabajo. Durante estos últimos 30 años, siempre ha existido el interés de nativos y de instituciones de apoyo, de mejorar este proceso de tratamiento de la paja toquilla con el fin de expender un producto terminado con excelente propiedades y calidad y siempre será constante de intervenciones con el fin de apoyar una actividad que genera la materia prima para la confección de los famosos sombreros de exportación.
Este trabajo de investigación, innovación y mejoras, aporta a las iniciativas de seguir tecnificando la actividad artesanal del tratamiento de la paja toquilla.

\section{Materiales y Métodos}

\subsection{Programa de producción y su control.}

El programa de producción debe basarse en el diseño de producción trabajado porque debe partir de un tiempo estándar para una unidad productiva (tiempo unitario de producción, TUP). Así, la investigación y el estudio de los métodos y tiempos en cada parte del proceso nos lleva a definir el tiempo unitario de producción o cuanto producimos en una hora de trabajo. De acuerdo a ello, se debe programar el trabajo para las horas necesarias y requeridas de producir: cantidades/hora, cantidades/día, cantidades/semana, cantidades/meses; cantidades/año. Ahora bien, el programa de producción, no solo es de las cantidades de productos, sino también, de los recursos humanos, de las maquinarias y equipos y de otros recursos que intervienen en la producción. Aquí se habla de los turnos de trabajo hasta completar lo programado. Por ejemplo, un proyecto o empresa, puede tener un solo turno de trabajo de 8 horas y en ese lapso de tiempo, producir unas 300 unidades, como resultado del diseño del sistema de producción (métodos y tiempos de trabajo básico). Entonces, en un día de trabajo de 24 horas se producirá 900 unidades y si se trabaja 7 días a la semana, 4 semanas al mes y 12 meses al año, entonces la programación de la producción anual estará basada en 302400 unidades. Esta cuantificación básica servirá para la programación de los otros recursos como son personas, maquinarias, suministros y la relación de disponibilidad en stock.

\subsection{El sistema de producción del Centro artesanal de procesamiento de paja toquilla Barcelona. Descripción.}

Para poder definir este tipo de sistema de trabajo, debemos aclarar que el trabajo realizado hasta ahora es eminentemente artesanal apegado a un sistema de producción por proceso. Estos son:

- Limpiar y formar manojo

- Cocinado

- Secado 
- Blanqueado

- Empacado

El sistema de producción adoptado por el Centro Artesanal de Procesamiento de Paja Toquilla en la Comuna Barcelona, es por PROCESOS CONTINUOS, o sea, procesos artesanales o manuales que dependen unos de otros para la continuidad y llegar al producto final.

La materia prima inicial es el COGOLLO cortado de la mata o planta silvestre en la montaña y que comienza a ser trabajado y tratado (cortado, limpiado, cocinado, secado y armado) para obtener un producto final que es LA PACA.

El sistema de producción en el Centro de tratamiento de paja toquilla de la Comuna Barcelona, comienza una vez que ha llegado la materia prima que es el conjunto 112 de tallos o cogollos llamado “ocho”. El tallo es la parte de la planta que es cortado una vez que ha crecido en una altura de unos dos metros aproximadamente y en unos 2 a 3 años. En la foto siguiente se muestra un “ocho”, listo para su procesamiento.

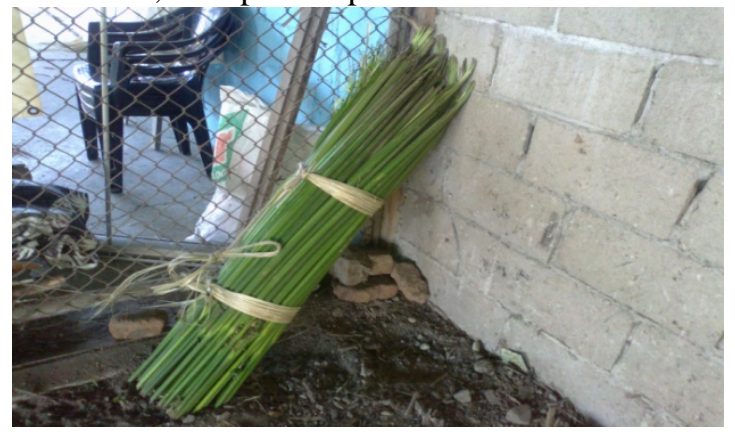

Fig. 1. Un "Ocho"

Fuente: Área Almacenaje. Centro Artesanal

Entonces, comienza el proceso con el DESCONCHADO.

Este proceso consiste en abrir cada tallo del "ocho" (que esta rígidamente unido desde que llega) y sacarle la corteza externa y abrirlas en hoja. Esta es una actividad que se la hace manualmente y tiene un tiempo de duración de entre 25 a 30 minutos, dependiendo de la habilidad de la persona

\section{EL ESPINADO}

Consiste en abrir las hojas de cada tallo con una agujeta en su parte final, desde unos 20 centímetros para dejarla lista a desprenderla. De igual manera, esta actividad dura entre 25 a 30 minutos, dependiendo de la habilidad de la persona.

SACADO Y LIMPIADO

Aprovechando las hojas espinadas, se hace la limpieza del tallo dejándolo libre, sacándole hojas que no entran al proceso de cocinado. Queda así formado el cogollo para la formación de "reales". FORMACION DE “REALES"

Con 14 "cogollos" limpios y listos, se forman un "real” uniéndolos y asegurándolos con hilosresiduos de la misma paja. Esta actividad dura 10 minutos.

Hasta aquí, este trabajo lo realiza una mujer que son las personas hábiles en estas actividades. De allí, el siguiente paso consiste en que una persona, hombre y que es el encargado de cocinar, realice la actividad de corte del tallo del real en su parte denominada cabeza.

EI CORTE DE CABEZA DEL “REAL”, se lo hace apoyado en una estaca de madera y con un machete, el operario, corta las cabezas de los tallos del "real" en unos 10 centímetros. Estas generalmente cuando se forma el real tiene una extensión de unos 25 centímetros. Su corte es para minimizar el largo del tallo y mejorar la maniobra presentando un tallo con distancia de unos 12 a 15 centímetros. Esta actividad también puede durar unos 10 minutos. La siguiente foto muestra el corte de la cabeza.

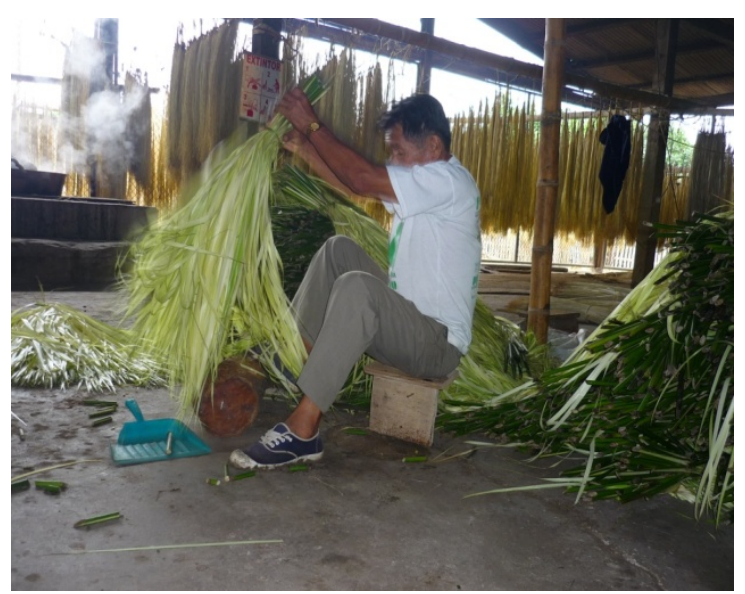

Fig. 2. Operario cogiendo el "real” para proceder al corte de la cabeza.

Fuente: Área procesamiento. Centro Artesanal

\section{PREPARADO DE LA PAILA}

Para poder cocinar la paja (reales formados), se utiliza 3 hornos de cocinada. Cada horno forma un hogar en un área de aproximadamente 5,25 $\mathrm{m}^{2} \mathrm{y}$ que consiste una estructura base en el piso de ladrillos refractarios sobre el que descansa una plancha de acero, que es soporte de la paila y dejando el espacio inferior necesario para la combustión que se alimenta con leña. La paila es un recipiente de bronce. Formada así los hornos, se llena la paila con agua por aproximadamente 10 galones y se enciende la llama y se espera que en 
45 minutos este hirviendo para agregar y cocinar la paja.

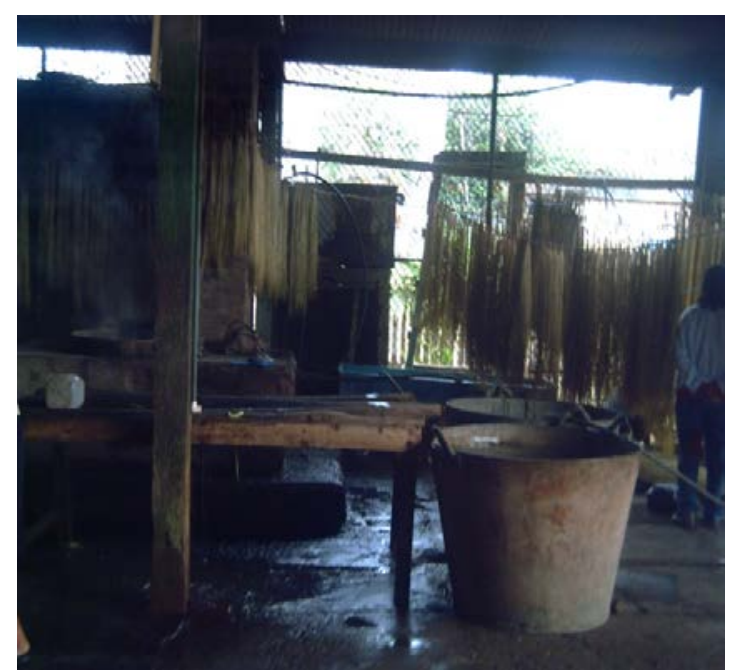

Fig. 3. Paila de bronce

Fuente: Área Cocción. Centro Artesanal

\section{COCINADO}

Cuando el agua esta lista hirviendo, se introduce a la paila los "reales" de acuerdo a su capacidad que generalmente es 36 "reales" o 4,5 "ochos" que se cocina en aproximadamente 45 minutos. Durante este tiempo, las personas encargadas de esta actividad, mueven los reales introducidos en la paila con el fin de homogenizar el cocinado.

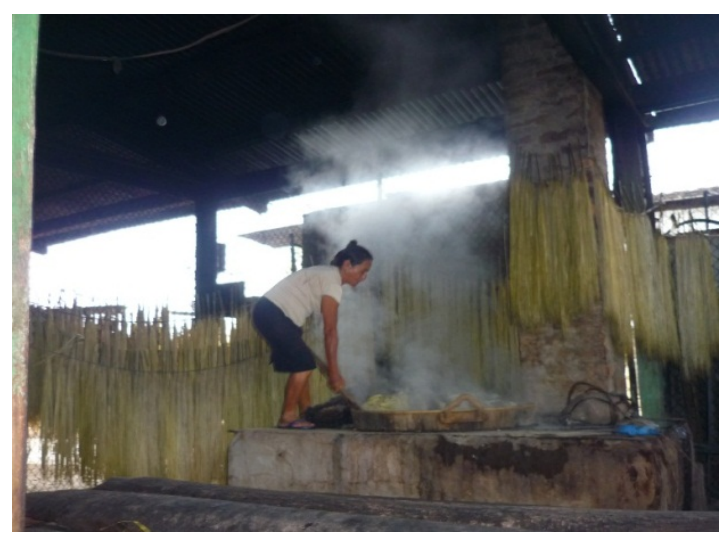

Fig. 4. Cocinado con Leña Fuente: ÂreaCocción. Centro Artesanal

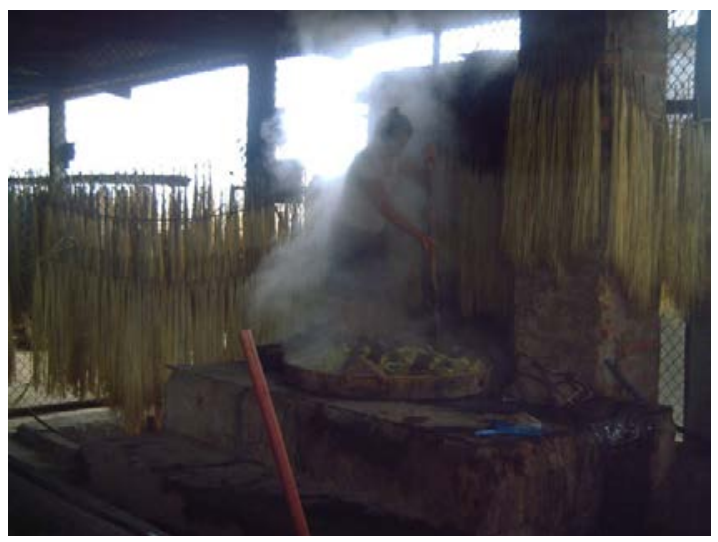

Fig. 5. Cocción

Fuente: Área Cocción. Centro Artesanal

\section{SACADO Y ESCURRIDO EN MESA DE} TRABAJO

Una vez transcurrido el tiempo de cocinado, Se saca el producto cocinado en mesa de trabajo para que escurra el agua y baje la temperatura y humedad al medio ambiente. El tiempo estimado es de unos 10 a 15 minutos. Para secar la paja cocinada, se utiliza unos ganchos de madera. Antes de trasladar al área de secado y en la medida que se va cargando los "reales" a una carreta, se los sacude con el fin de sacar la humedad.

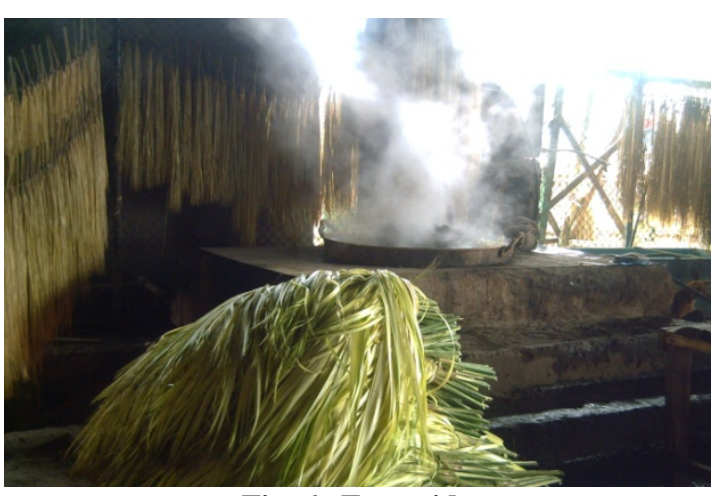

Fig. 6. Escurrido

Fuente: Área Cocción. Centro Artesanal

\section{TRANSPORTE Y SECADO}

En una carreta o triciclo se coloca lo que ha sido cocinado y se traslada al área de secado en donde se va a tender. Este tiempo es aproximadamente 15 minutos y corresponde prácticamente al tiempo de secado. 


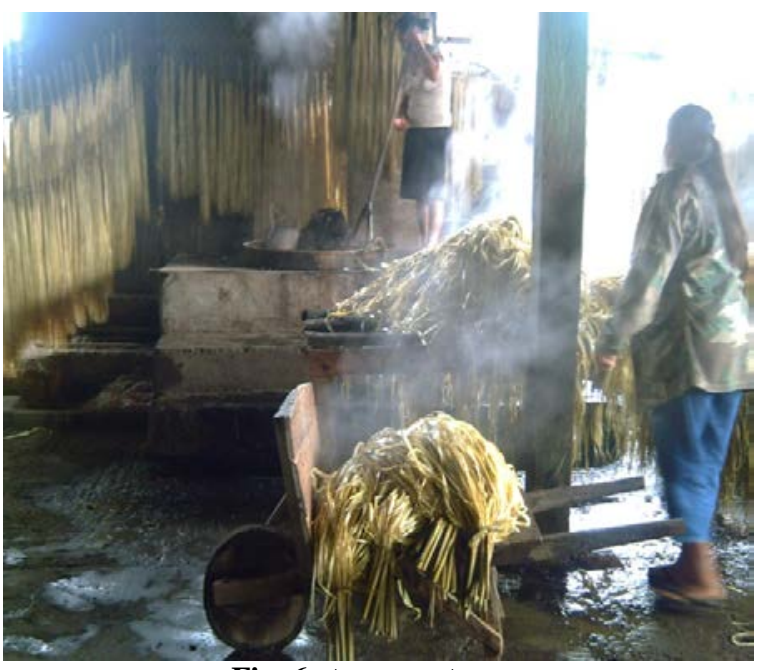

Fig. 6. transporte

Fuente: Área Cocción. Centro Artesanal

\section{TENDIDA}

En 25 minutos se hace la tendida que consiste en desatar los reales y tender los cogollos individuales. Este tiempo ya corresponde al tiempo inicial de la secada al ambiente y para ello, la planta tiene un área de secado.

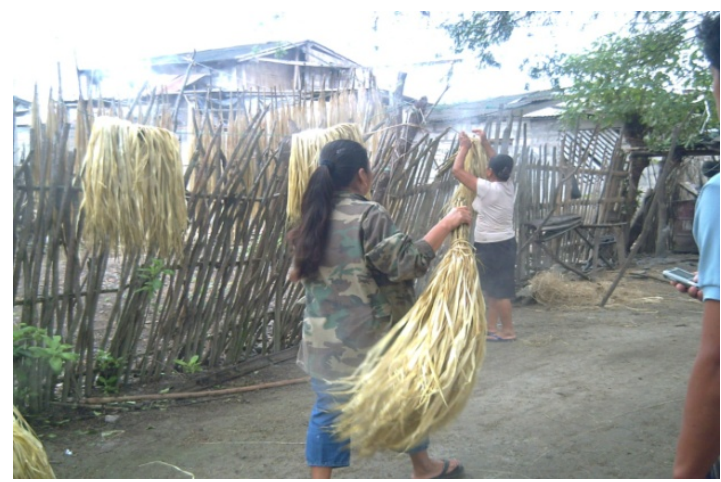

Fig. 7. Tendido

Fuente: Área Secado. Centro Artesanal

\section{SECADO}

Al medio ambiente. Si el ambiente está nublado, puede secar bajo techo en 12 horas. Si hay sol, entonces puede secar bajo sombra 3 horas y de allí dos horas al sol.

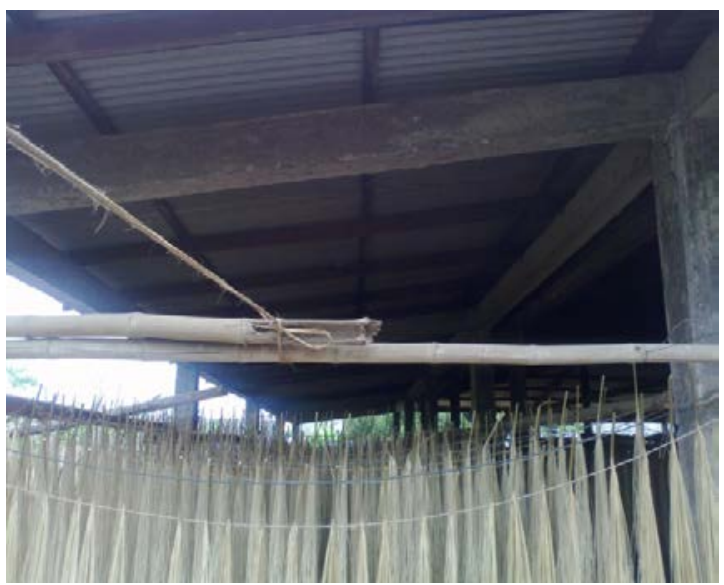

Fig. 8. Secado natural

Fuente: Área Secado. Centro Artesanal

\section{RECOGIDA Y FORMACION DE PACA}

Una vez, secado el producto, se recoge y comienza la formación de pacas, las misma que es la unión de 28 "ochos". Estos son asegurados con sacos de polietileno, están listos para entregar.

Es de considerar que en todo este proceso es generalmente descrito para la formación y cocción de un "ocho". Si se considera que se desea determinar el tiempo de una paca entonces el tiempo debe ser en la formación de una paca.

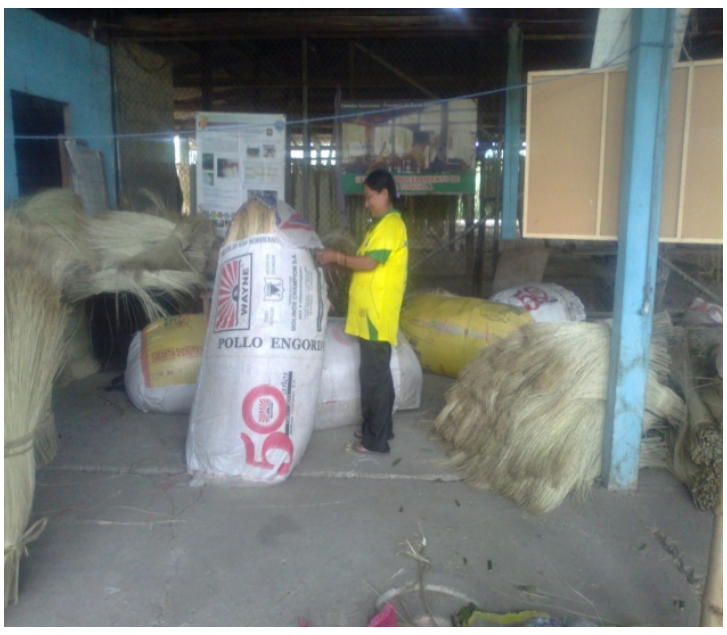

Fig. 9. Empacado

Fuente: Área Empacado. Centro Artesanal

\subsection{Tiempos de producción de una paca como producto final en secado al ambiente.}

Para poder producir una PACA como producto final, se toma en consideración tiempos unitarios de cada actividad. También se considera que en las diferentes áreas del proceso y de acuerdo a la 
disponibilidad de las 4 cocinas, un total de 10 mujeres en las primeras 4 actividades como son:

- Desconchado

- Espinado

- Sacado y Limpiado

- $\quad$ Formación de reales

Se considera un total de personas 2 varones y 2 mujeres en los procesos de:

- Preparar agua a la paila y encender el quemador al horno.

- Corte de cabeza de tallo

- Cocinada

- Sacada y sacudida y tendida para secado

- Secado al medio ambiente

- Recogida y formada de la paca

Esto da un total de 1460 minutos o sea 24, 33 horas

\section{RESULTADOS}

3.1. Mejoras en los sistemas de producción y secado.

3.1.1. Construcción e instalación de nuevos quemadores.

Para mejorar el proceso de cocinado, eliminando el anterior que se utilizaba leña y residuos de madera como combustible y que contaminaba el ambiente, se construyeron y se instalaron quemadores para la utilización de gas licuado de petróleo, GLP. Se instalaron en cada horno (3) cañerías de alimentación desde el tanque al quemador, dándole las seguridades del caso. En el siguiente grafico están las pruebas del quemador construido y su funcionamiento en uno de los hornos. El quemador, está construido de un tubo de acero al carbono de 6 pulgadas de diámetro y 50 centímetros. En su interior está alojado el sistema de una boquilla que se alimenta de mangueras a un tanque de gas de $14 \mathrm{Kg}$, el mismo que tiene sus válvulas de control. El sistema de mangueras está constituido con manguera de $1 / 2$ pulgadas y llaves de paso y presión. Secadores de estas características fueron instalados para las cámaras de secado.

$\mathrm{Al}$ encender la llama, se utiliza un quemador con conexiones a una fuente de combustible, en este caso, GAS LICUADO DE PETROLEO, GLP.

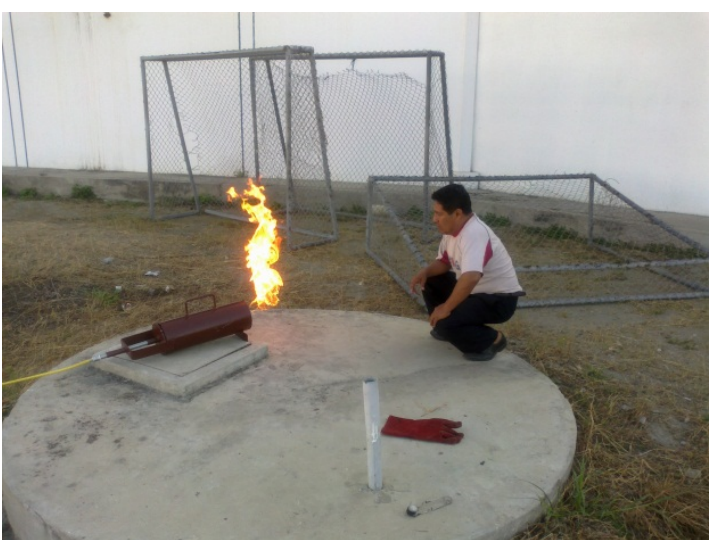

Fig. 10. Prueba de quemadores a G.L.P.

Fuente: Taller Industrial. Universidad Estatal Península de Santa Elena. UPSE

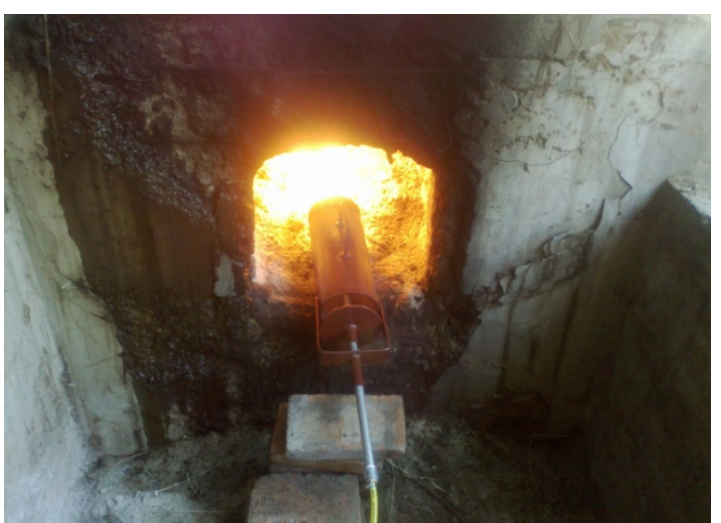

Fig. 11. Combustión de quemadores a G.L.P. Fuente: Área de Cocción. Centro Artesanal.

\subsubsection{Construcción de Secadores alternativos}

Se ha construidos dos secadores en un área escogida que comprende una longitud de $18 \mathrm{~m}$. por 3,3 m., de ancho y por 2,60 m., de alto constituyendo un volumen de 154,4 metros cúbicos, los cuales se subdividen en dos áreas para la formación de dos secadores juntos. Las paredes de estos secadores están construidas de bloque de arcilla, teniendo sus puertas de ingreso soportadas en el pilar que divide ambas áreas. Los ductos internos para la distribución de la temperatura dentro de cada uno de los secadores, están constituidos por 3 ductos en la parte interior y superior a lo largo del área en cada secador. Estos ductos $(0,35 \times 0,12 \times 8 \mathrm{~m}$.) están construidos de lámina de acero galvanizado y se sostiene de la infraestructura de las vigas del techado. En la parte externa-lateral, de toda esta infraestructura, se colocan el sistema de generación de calor que consiste en ubicación del blower (1HP; 110/220 
v; 60/50 Hz 10,7 Am; 3340/2280 RPM; 42/36 $\mathrm{m}^{3} /$ minutos) y quemador a una altura aproximada de 2,20 m., soportado por estructura base de metal. Se realizan las conexiones eléctricas respectivas para activar el blower por medio de contactores y botoneras y estas a su vez, alimentadas por una nueva instalación de acometida hasta este lugar.

La capacidad de cada secador es de entre 12 a 14 "ochos" (1568 tallos) en un promedio de 2,5 horas.

\subsection{Tiempos de producción de una paca como producto final con secadores construidos}

El tiempo total $\mathrm{y}$ siguiendo las mismas consideraciones de numero de cocinas, número de personas, cuando se hacen las mismas actividades hasta llevar a los secadores, entonces el tiempo de elaboración de una paca es de 500 minutos los que da un tiempo unitario de 500 minutos o sea 8,33 horas o un día de trabajo.

$\mathrm{Si}$ se trabajan en 2 turnos continuos $\mathrm{y}$ con secadores industriales, se estarían elaborando 2 pacas/día y si trabajamos 25 días/mes, entonces, se elaborarían 50 pacas. En cambio se trabajan bajo secado ambiente se elaborarían una paca día o sea 25 pacas/mes

El gráfico adjunto muestras los tiempos y la diferencias de ambos procesos.

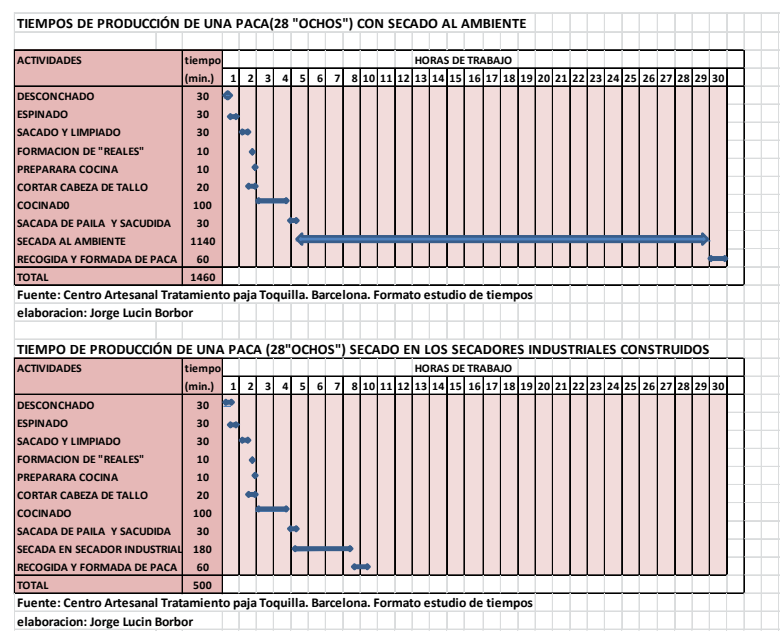

Fig. 12. Tiempos de procesos de tratamiento Fuente: Formatos de estudios de tiempos-métodos

\subsection{Procedimiento para el funcionamiento de los secadores industriales}

\section{a. Alimentación de la paja}

En el interior de cada secador, se deben disponer los cogollos, tendidos en cordeles en el sentido longitudinal, con espacios aproximados de 12 centímetros, para poder llenar la capacidad. Previamente, luego del cocinado, estos son escurridos y sacudidos al medio ambiente lo que logra bajar la temperatura y humedad.

\section{b. encendido de la fuente de calor}

Primeramente se debe colocar el tanque de gas en sentido normal, nunca acostado ni inclinado. La salida de gas hacia el quemador (despresurización) y el contacto al ambiente, causa que, el área de contacto del tanque con el medio ambiente se enfrié a bajas temperatura y muchas veces ir a congelamiento externo. Para evitar esto se coloca el tanque en una lavacara con agua. Este intercambio de temperatura en la despresurización no acarrea peligro.

Se debe abrir, primeramente la válvula de paso que está entre el quemador y el tanque de gas conectado por manguera especial. Esta apertura es solo al 1/8 de su apertura total.

Luego se debe abrir la llave de presión que está situado sobre el tanque, de una manera lenta y con el propósito de que pase el gas a la boquilla del quemador de una manera momentánea cerrándole inmediatamente. Con el chispeador, la persona encargada debe accionarlo en la boquilla del quemador, que inmediatamente se enciende en virtud de que ha llegado y quedado gas. Encendido el quemador, se regula la alimentación de gas en la válvula de presión para que suba la llama en una longitud aproximada de 35 centímetros. Inmediatamente, se activa el breaker de alimentación de energía eléctrica y se pulsa el botón verde de encendido del panel de control. El blower comienza a funcionar, atrayendo la llama para introducir el calor al secador. La regulación de la llama debe ser tal que no llegue con fuerza y cause ruidos al sistema del blower.

Cuando sucede esto, se debe bajar la apertura de la válvula de presión.

\section{c. control de secado.}

En el interior, debe existir una temperatura media entre 40 a 45 grados centígrados. Cada 20 minutos, la persona encargada de secador debe apagarlo con el fin de alternar y mover los cogollos a las zonas de mayor ventilación para buscar el secado necesario de todo el conjunto. Luego de esto, inmediatamente lo prende nuevamente y continúa el trabajo del secador. 
Alternadamente, tiene que ingresar la persona para verificar el proceso de secado.

La Capacidad de cada secador es de aproximadamente 12 a 14 "ochos" que se secan alternadamente en una 2 a 2,5 horas. Luego de haber eliminado la humedad, los cogollos se sacan y se tienden a la intemperie en el área de secado normal, por unas dos horas, logrando el objetivo final.

Para llevar un control de esta actividad, se utiliza el formato CONTROL DEL PROCESO DE SECADO, que debe aplicárselo y hacer una evaluación final mensual

En el formato la hora inicial, corresponde a la hora en que se activa el sistema de secado (quemadorblower), y la hora final cuando se ha terminado de secar toda la capacidad del secador. La cantidad debe ser cuantificada en "ochos”. El tiempo total de secado es en minutos u horas haciendo la transformación. La temperatura alcanzada está entre 40 a 45 grados centígrados. El consumo de gas es en unidades de tanque o en kilogramos. Para ello es importante relacionar el proceso de secado y la cantidad de gas y el tiempo utilizado en los procesos. Se pudo haber secado 12 "ochos" en el tiempo requerido y todavía existe gas en el tanque para otro proceso. Este nuevo proceso puede consumir el sobrante hasta un determinado tiempo. Allí es preciso hacer el cálculo. El estado de secado se lo valora como poco aceptable, aceptable, muy aceptable.

El fin de mes se debe hacer una evaluación de estos parámetros

\section{MEDIDAS DE SEGURIDAD}

- Abrir las válvulas de paso y presión de acuerdo a lo indicado.

- Nunca acostar ni inclinar el tanque de gas

- Utilizar el chispeador para generar la llama en la boquilla del quemador.

- Utilizar una escalera para subir hasta la boquilla del quemador

- Al accionar/apagar el breaker y la botonera de encendido del blower, nunca ir con las manos ni el cuerpo húmedo peor mojado.

- Al apagar el secador para los cambios internos de la paja, abrir y esperar un momento antes de ingresar para que salga un poco la temperatura. Tener cuidado de ingresar cuando se esté secando producto. Si se necesita una verificación tener cuidado con los cambios de temperatura. Solo personas en estado de salud normal pueden hacer esta actividad.

\subsection{Diagrama de las operaciones del proceso}

El diagrama de operaciones del proceso es una hoja técnica mostrando la secuencia de producción con el método propuesto de mejoras. Se adjunta el diagrama que muestra la secuencia productiva

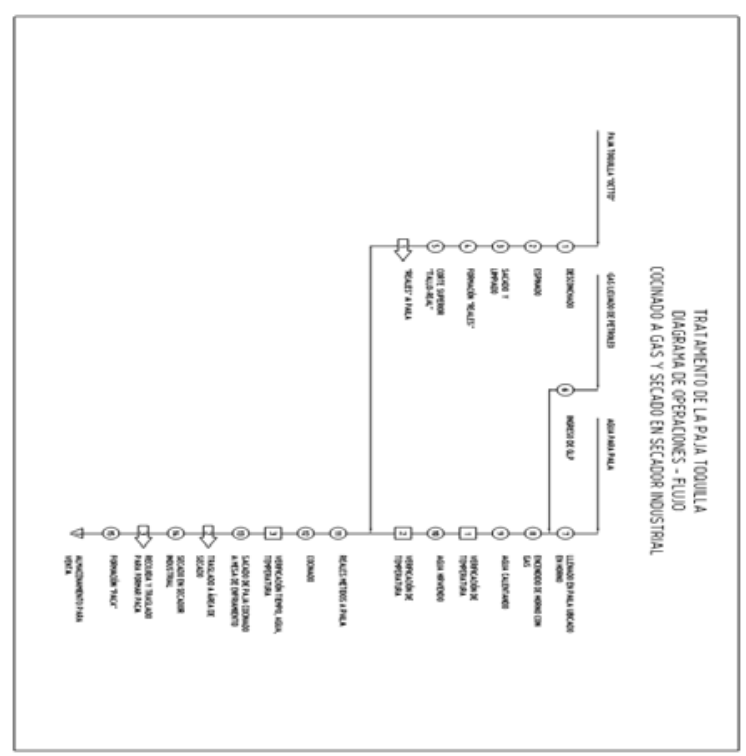

Figura 13: Diagrama de operaciones del proceso.

Fuente: formatos métodos de trabajo

\subsection{Análisis de costos de producción}

Uno de los aspectos importantes de los proyectos de transformación de materias primas, es determinar los costos de la producción que se incurre para obtener el producto. Cada proyecto de acuerdo a su naturaleza tiene sus propios costos de producción de acuerdo a un programa de producción establecido en la fase técnica y es necesario determinarlos logrando un balance de necesidades que logre minimizarlos.

Los costos de producción están formado por:

- Materias primas o Materiales Directos

- Mano de Obra directa

- Gastos Fabriles

costos de materias primas o materiales directos.

Corresponde al valor que se incurre en la compra de los materiales que conforman directamente el 
producto y que son trasformados por las maquinarias del sistema productivo.

En el Centro de tratamiento de paja toquilla de Barcelona las materias primas que ingresan a transformarse son:

- la paja toquilla y que es comprada como unidad de medida de "un ocho" y cuyo valor puesto en sitio de producción es de \$ 6. Para producir una paca que es el producto final a venderse se deben utilizar 28 "ochos" o sea, el costo será $\$ 168$

- El agua que se alimenta en cada paila se considera materia prima porque cuando la paja es cocinada, influye en la trasformación del producto dándole resistencia, dureza y solidez. Para cocinar una paca, se necesitan 28 “ochos” y entonces el costo \$ 0,0392 por agua.

El costo de mano de obra directa

Representa los costos-pago de la mano de obra que directamente interviene en el proceso de trasformación de las materias primas y normalmente tiene una responsabilidad en el manejo de maquinarias directas. La asignación de este costo depende de las disposiciones legales de cada país. En nuestro país, Ecuador, actualmente reciben su remuneración establecida en ley a un salario mínimo vital más las compensaciones de ley (aproximadamente un 36,16\% del salario básico de \$340) totalizando unos 463 dólares/mes.

Para el caso del Centro, la mano de obra se paga por la limpieza y cocinada. La Limpieza se paga \$ 1 y por la cocinada, \$ 1,5. Para la producción de una paga o sea 28 "ochos", se paga \$ 28 por la limpieza y \$ 42 por la cocinada

Los costos y gastos de fabricación.

Los costos de fabricación, son aquellos que sirven para producir el bien y que están ligados a la actividad productiva de trasformación de una manera indirecta Se subdividen en:

- Mano de obra indirecta

- Materiales Indirectos de producción

- Mantenimiento en general

- Depreciaciones de maquinarias, equipos y herramental de producción

- Suministros y Materiales energéticos para producción

- Seguros en general.

- Imprevistos
A. mano de obra indirecta. Este rubro tiene que ver con el coste de los salarios y remuneraciones de las personas que de una manera indirecta tienen que ver con la producción. Así tenemos que de este rubro dependen, los jefes, supervisores e inspectores y trabajadores de producción, de control de calidad, de seguridad industrial, de mantenimiento, de medio ambiente, de proyectos investigativos. A ellos, deben pagársele lo que de acuerdo a la ley, experiencia, profesionalidad, competencias, deban ganar para cumplir determinados requisitos en cada puesto.

En el Centro de Tratamiento paja toquilla, no se paga este costo.

B. materiales indirectos de producción. En este rubro debe costearse, comprar y pagar, aquellos materiales que no forman parte sustancial-directa del producto, pero que para su presentación, traslado, comodidad, son necesarios en el proceso de producción. Este concepto implica entender que este material, no es transformado por el sistema de producción que se propone. Así, tenemos los empaques, cartones, aditamentos, seguridades externas de amarre de catones, soportes-pallet y otros.

En el Centro de Tratamiento, los materiales indirectos son:

- El saco para envolver la paca y que se requieren unos 3 sacos de yute y cuyo costo es de $\$ 0,24$

- La piola para asegurar la paca envuelta y que se utiliza unos 3 metros con un costo de $\$ 0,075$

C. suministros y materiales energéticos de producción. Aquí se costea la energía eléctrica, combustibles, agua, otro tipo de energía que sirve para apoyar al sistema de producción. Se realiza un cálculo de la cantidad incurrida en el mes, basadas en el programa de producción y se multiplica por el costo unitario industrial proyectándolo anualmente. Medida de ejemplo, y en consumo de energía eléctrica en cada país, existe codificaciones de pago por consumo de Kilovatios/hora. En Ecuador, de acuerdo 
a las políticas gubernamentales se establecen rango de costos por KW/h.

En el Centro de paja toquilla se costea como suministros industriales

- $\quad$ Por luz se paga $\$ 0,25$ por cada paca y por gas se paga $\$ 3$ por la cocinada y $\$$ 3 por la secada en el secador industrial para el secado de una paca.

D. el mantenimiento. Aquí se costea los materiales, suministros, trabajos (aceite, lubricantes, repuestos, materiales de limpieza) que sirven para el mantenimiento, reparación y conservación de las maquinarias y equipo de producción y en general de la empresa. Es interesante definir este concepto desde el punto de vista del trabajo de la maquinaria. Cuando es nueva existe poca frecuencia en el mantenimiento pero, en la medida que su trabajo es mayor con el pasar del tiempo, la necesidad de mantenimiento es mayor.

No se estipula un costo de mantenimiento por la paca

E. Las depreciaciones Depreciación y Obsolescencia. En este rubro debe costearse la depreciación de los activos fijos en los que se ha invertido en el proyecto, excepto el terreno y que forman parte de los activos en relación directa con el proceso productivo. Es importante señalar que la pérdida del valor económico y físicos/desgaste de los activos toma el nombre de depreciación y obsolescencia. En el primer caso, el activo pierde su valor porque la tecnología y ciencia lo reemplaza con un moderno prototipo y en el segundo caso el valor de la inversión obedece a que con el avance del tiempo, la capacidad de trabajo disminuye y los costos por el mantenimiento, llegan a un total que es imposible económicamente mantenerlo. La asociación de ambos conceptos nos lleva a asignar un valor por depreciación y obsolescencia. Este valor considera al activo renovándolo, calculando para el efecto, el valor anual de acuerdo a la asignación de su vida útil. Esta depreciación, es llamada "insumo físico" ya que representa el costo imponderable anual del activo que se trasforma en insumo para producir un bien y se asocia en el costo fabril. Pero a su vez, este insumo se convierte en un acervo paralelo renovable o recuperable para no disminuir la inversión inicial.

Para la paca se ha considerado la construcción de los secadores industriales, la depreciación y su costo es $\$ 4,16$.

En el siguiente cuadro, se muestra los costos de producción calculados y desglosados

Tabla 1: Costos de producción

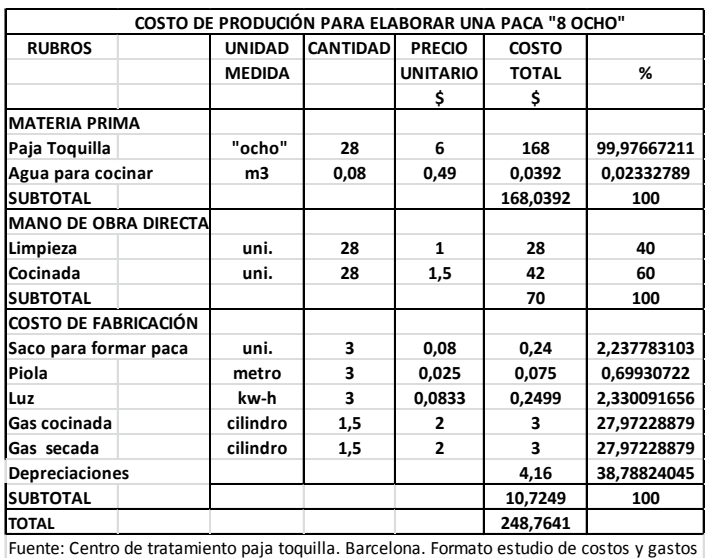
Elaboración: Jorge Lucín Borbor

El costo total de producción de una paca será de \$ 248,7641. De este costo, la materia prima tiene un total de \$168,0392 y aporta un $67,55 \%$. La mano de obra directa aporta con un 28,14\% y totaliza \$ 70 y los gastos de fabricación hacen \$ 10,7249 y aportan un $4,31 \%$.

El costo total operativo de la producción de una paca.

Por otro lado es de resaltar que no existen gastos administrativos y que el movimiento para la venta de las pacas se toma como un GASTO DE VENTA y que para el traslado de la paca al Azuay, se asocia un costo por trasporte, viáticos y estadía de \$ 4.

Esto implica sumar al costo de producción el gasto de venta y entonces, tenemos el COSTO OPERATIVO TOTAL DE LA PACA que es de $\$ 252,7641$ 


\subsection{Análisis de rentabilidad}

Para la venta de la Paca se tiene un precio de venta de $\$ 280$, entonces queda una rentabilidad de

Tabla 2. Rentabilidad

\begin{tabular}{|l|l|ll|}
\hline Rentabilidad & $\begin{array}{l}\text { Precio de } \\
\text { venta }\end{array}$ & $\begin{array}{l}\text { Costos } \\
\text { Gastos }\end{array}$ & y \\
\hline \$27,2359 & $\$ 280$ & & \$252,7641 \\
\hline
\end{tabular}

Fuente: estudio de costos del Centro Tratamiento

\subsection{Distribución de áreas del Centro Artesanal de procesamiento de paja toquilla}

En el siguiente gráfico, se puede observar, las áreas que conforman el Centro de procesamiento de paja toquilla en la Comuna Barcelona. El total de área útil para el trabajo es de 1249,3 metros cuadrado.

Las principales áreas son:

- $\quad$ Áreas de secado natural de $171 \mathrm{~m}^{2}$

- $\quad$ Área de secado natural 342,25 m. ${ }^{2}$

- $\quad$ Área de cocinado de 98 m. ${ }^{2}$

- Área de secador industrial $1 \mathrm{de} 54 \mathrm{~m}^{2}$

- Área de secador industrial 2 de $25 \mathrm{~m}^{2}{ }^{2}$

- Área de oficina y bodega de $68 \mathrm{~m}^{2}$

Se adjunta el diagrama que muestra las áreas

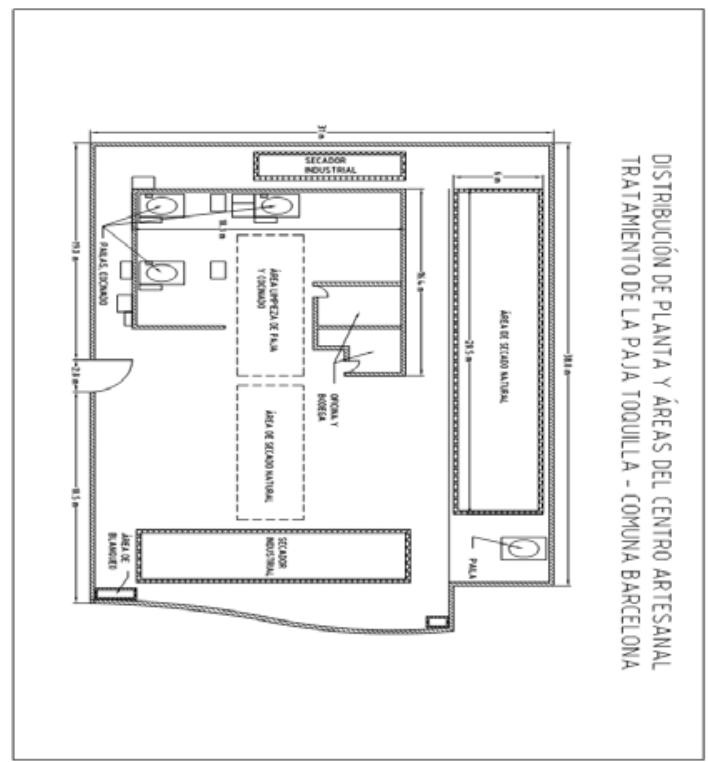

Figura 14: Diagrama de Distribución de áreas Fuente: Sitio. Centro Tratamiento
Si se producen unas 35 pacas en el mes que se pueden entregar a los clientes, entonces la rentabilidad mensual será de \$953,2565 que representa anualmente \$11439.

En función del nuevo trabajo de las cámaras de secado, la producción de 50 pacas al mes, generaría una rentabilidad mensual de \$1361,80 y \$16341,54 anuales.

\section{Conclusiones}

Se determinan las siguientes conclusiones:

1. Se organiza la gestión administrativa y técnica basado en formatos diseñados que permiten el control de las actividades

2. El cambio de la utilización de combustible eliminando la leña por el alternativo de GLP, permite la disminución sustancial del impacto a la contaminación ambiental y evita el deterioro del bosque al cortar árboles.

3. La utilización de nuevos secadores industriales, agiliza el tiempo de secado, elimina cuellos de botellas en la relación producción-secado y mejora la calidad de la fibra y contribuyendo al aprovechamiento del tiempo en el proceso general del tratamiento.

4. Con el estudio de métodos de trabajo y tiempos, se busca que el proceso sea controlado eficientemente en sus etapas con los reportes técnicos de las actividades $\mathrm{y}$

5. Esto a su vez, permite definir los costos que se producen en este proceso de tratamiento determinando las rentabilidades, que si bien pueden ser más atractivas, sirven para proyectar el aumento del precio unitario de venta en función del mejoramiento de los procesos y de la calidad del producto.

Una posibilidad de ello, es recomendada y debe darse para justificar los recursos invertidos en un trabajo que muestra aportes profesionales tanto de la comunidad, de los aportantes, así como de las consultorías. 


\section{Agradecimientos.}

A José García. Grupo NOBIS

A Alexandra Jara Escobar. MSc. Revisión

Abstract

\section{Bibliografía.}

[1]Nievel Benjamín, FreivaldsAndris. 2010. Ingeniería Industrial. Métodos estándares y diseño de trabajo. McGraaw Hill.

[2]Gary Dessler. Administración de Recursos Humanos.2009. Pearson Prentice Hall

[3] Creus Antonio (2011). Instrumentación Industrial.

[4] Álvarez Heredia Francisco; Faizal Geageas Enriqueta. 2012. Riesgos Laborales. Como prevenirlos en el ámbito de trabajo. Ediciones de la U.

[5] Pacheco Chavira Jesús Neri. 2010. Mediciones y Control de Procesos Industriales. Ediciones Trillas.

[6] Carpintero Pino José María. 2013. Soldadura Básica. Guía Práctica. Starbook

[7] R.C. Hibbeler. Mecánica de materiales. 2010. Pearson.

[8]. López Rodríguez Ricardo 2011. Mecánica. Ediciones Tabor.

[9] Daniel Polit Arguello, Marcos Buestán Benavides. 2006. Establecimientos de Parámetros de diseño para el proceso de secado de paja toquilla por medio de la metodología de diseño para seis sigmas. ESPOL.

[10] HeizrRender. 2005. Principio de Administración de Operaciones. Pearson Prentice Hall.

[11] A. Boero, J. Duque.2008. Sistema de Calentamiento de Aire y Cámara de Secado para Paja Toquilla por Recuperación de Energía desde los Hornos Artesanales de Cocción de la Paja. Revista Tecnología ESPOL.

[12]. Luis Torres Rodríguez. 2004. Creación y formalización de la microempresa civil. Publicación de Fundación Avanzar.

[13] Grundfos. Manual del instalador. Sistemas de calefacción de vivienda. 2008. www.grundfos.gob

[15] INEC. Censo nacional de Población y Vivienda 2010. www.inec.gob.ec.

[16] Consorcio Nobis. FundaciónNobis.Revista empresarial del grupo Nobis. Responsabilidad social. Febrero 2015.www.consorcionobis.com.ec [17]Hargadon. Contabilidad de costos. [18]LelandBlank. Antony Tarquín. Ingeniería económica.2012.McGraw Hill.
[19] Nassir Sapag. Chaín. 2012. Proyectos de Inversión Formulación y evaluación. Pearson Prentice Hall.

[20] Gerardo Guajardo Cantú. Nora Andrade.2008 Contabilidad financiera. Mc Graw Hill.

[21] Raúl Vilcarromero Ruiz. La gestión en la Producción. 2013..http://www.eumed.net/librosgratis/2013a/1321/control.html.

[22]JayHeizer. Barry Render. 2006. Dirección de la producción. Pearson. Prentice Hall.

[23]RenderHeizar. Administración de operaciones. 2010. Pearson. Prentice Hall.

[24]. El sombrero de paja toquilla, patrimonio Cultural Inmaterial de la humanidad. 2014.www.turismo.gob.ec.

[25]Besterfields. Control de calidad. 2004. Pearson. Prentice Hall.

[26] Gutiérrez Pulido. 2006. Calidad Total y productividad. Mc Graw Hill. 